\title{
Shared picture book reading interventions for child language development: a systematic review and meta-analysis
}

Article

Accepted Version

Dowdall, N., Melendez-Torres, G. J., Murray, L., Gardner, F., Hartford, L. and Cooper, P. J. (2020) Shared picture book reading interventions for child language development: a systematic review and meta-analysis. Child Development, 91 (2). e383-e399. ISSN 0009-3920 doi:

https://doi.org/10.1111/cdev.13225 Available at https://centaur.reading.ac.uk/80756/

It is advisable to refer to the publisher's version if you intend to cite from the work. See Guidance on citing.

To link to this article DOI: http://dx.doi.org/10.1111/cdev.13225

Publisher: Wiley

All outputs in CentAUR are protected by Intellectual Property Rights law, including copyright law. Copyright and IPR is retained by the creators or other copyright holders. Terms and conditions for use of this material are defined in the End User Agreement. 


\section{CentAUR}

Central Archive at the University of Reading

Reading's research outputs online 
Running head: BOOK-SHARING META-ANALYSIS

Child Development (2019) In Press

Shared picture book reading interventions for child language development: a systematic review and meta-analysis

Dowdall, N., Melendez-Torres, G. J., , Murray, L., Gardiner, F. , Harrtford, L. \& Cooper, P.J. 
BOOK-SHARING META-ANALYSIS

\begin{abstract}
Interventions that train parents to share picture books with children are seen as a strategy for supporting child language development. We conducted meta-analyses using robust variance estimation modeling on results from 19 RCTs $\left(\mathrm{N}_{\text {total }}=2,594 ; \mathrm{M}_{\text {childage }}=1-6\right.$ years). Overall, book-sharing interventions had a small sized effect on both expressive language $(d=0.41)$ and receptive language $(d=0.26)$. They had a large effect on caregiver book-sharing competence $(d=1.01)$. The impact of the intervention on child language was moderated by intervention dosage, with lower dosage associated with a minimal impact. Child age and caregiver education level were unrelated to child outcome. This review and meta-analysis confirms the promise of book-sharing interventions for enhancing and accelerating child language development.
\end{abstract}

Keywords: Book-sharing, meta-analysis, child language 


\section{Background}

For almost four decades, following the seminal work of Ninio and Bruner (1978), and framed within a Vygotskian approach (Vygotsky, 1978), researchers have investigated how sharing books with young children affects their development. This has been considered a particularly effective technique for scaffolding a young child's cognition and language (Van Kleeck, 1998), and indeed, it has been argued that children's picture books embody specific features that make them an especially powerful vehicle for language learning (Murray, 2014). Good picture books tend to present the essential features of an object or event, devoid of detail or elaboration, often in simple line drawings. They commonly do so repetitively, with minor variation, which facilitates acquisition of the concepts depicted. This acquisition is also supported by the fact that, unlike the fleeting presentations of real life phenomena (and in particular actions or emotions), in a picture book the infant can review and rehearse at their own pace. The pictures also provide an opportunity for more complex conceptual elaboration, such as reflection on the meaning of events and their causes, and the characters' intentions and perspectives (Murray, 2014). Notably, a number of early observational studies showed that, in comparison to other activities, such as play or meal times, during shared reading mothers tend to label objects more frequently and children are provided with more consistent and informative feedback (Snow \& Goldfield, 1983; Wells, 1985).

In spite of the considerable potential benefits of book-sharing, research points to a number of barriers preventing children and caregivers from engaging in this activity. Thus, in contexts of socio-economic adversity, families are less likely to have access to books and learning materials (Le Roux, 2012). Accordingly, in such contexts caregivers engage in substantially less shared reading with their children (Berkule, Dreyer, Huberman, Fierman, \& Mendelsohn, 2007; Cooper et al., 2014; Le Roux, 2012). This is important because metaanalytic data have shown that frequency of shared reading is a strong predictor of language 
BOOK-SHARING META-ANALYSIS

development (Bus, Van Ijzendoorn, \& Pellegrini, 1995). Further, parents do not always use the most effective techniques to support their child's development in the book-sharing context (Ninio, 1980; Weigel, Lowman, \& Martin, 2007). Notably, observational studies have shown that families experiencing high levels of adversity are less likely than more advantaged families to use a style of book-sharing associated with positive language outcomes (Berkule et al., 2007; Bornstein \& Putnick, 2012; El Moussaoui \& Braster, 2011; Van Kleeck, 1998). These socio-economic barriers raise the question of the extent to which an intervention designed to promote and enhance carer book-sharing skills in low SES contexts would be of benefit to child language development.

\section{Book-Sharing Interventions and the Basis for their Effectiveness}

The dialogic book-sharing interventions that have been developed are based on the Vygotskian principle (Vygotsky, 1930-1934/1978) that improvements in child cognition and language come about through contingent, structured interactions, pitched at the child's developmental level, in which the parent follows the child's cues and focus of interest and provides stimulation that enhances their child's experience of the environment. Whitehurst and colleagues $(1994 ; 1988)$ were the first to formalize a set of dialogic book-sharing techniques based on this principle. Importantly, what distinguishes these techniques from simply reading to a child is the interactive quality and use of evocative behaviors. This includes asking open-ended questions that extend beyond the content of the book, and providing encouragement and praise for child participation (Lonigan \& Whitehurst, 1998; Whitehurst et al., 1988). It also includes extensive pointing and labeling from the carer in response to the child's focus of interest, thereby helping the child to forge associations between the object of interest and the verbal label. Finally, interactive book-sharing facilitates caregiver engagement in 'decontextualized talk', where the discussion extends 


\section{BOOK-SHARING META-ANALYSIS}

beyond the content or pictures in the book to concepts that are novel and unfamiliar to the child (Lonigan \& Whitehurst, 1998).

Dialogic book-sharing interventions generally consist of a number of sessions where parents, either individually or in groups, are provided with guidance on how to share picture books effectively with their children. The process commonly involves introducing parents to a number of core techniques that involve the use of evocative strategies tailored to elicit a verbal response from the child - for example, more open 'what or where or who' questions, as opposed to more closed 'yes or no' questions (Whitehurst et al., 1988). Parents are also taught to use informative feedback in the form of expansions and modeling. Many programs include participatory role-play between participant and instructor, where good practice is demonstrated and modeled. Intervention programs also often provide accompanying materials such as summary sheets containing key lessons for parents to take home to use as reminders (Arnold, Lonigan, Whitehurst, \& Epstein, 1994). Many programs also provide picture books for participating caregivers to take home.

\section{Book-Sharing Interventions: Setting, Delivery, Format and Location}

Book-sharing interventions have been carried out in a wide variety of settings, from community centers, to pre-school halls and participants' homes. One prominent setting has been in and around clinics or pediatric rooms. The rationale for including book-sharing interventions in pediatric settings has been to encourage new parents to adopt shared reading habits early in the lives of their children. There is considerable potential for reach and scale with this particular programme delivery model, particularly in countries with well-established universal obstetric and pediatric services. Typically, programme sites create a space in waiting rooms for parents and trained volunteers to read aloud to young children. This provides the opportunity for parents to be mentored in interactive shared reading techniques (Mendelsohn et al., 2001). One iteration of this model is the Video Interaction Project that 


\section{BOOK-SHARING META-ANALYSIS}

films mothers reading to children and then provides constructive feedback and materials for them to continue these activities at home (Mendelsohn et al., 2011). A small number of controlled studies evaluating video based programs have shown success in improving the expressive and receptive vocabularies of intervention children compared to controls (High, LaGasse, Becker, Ahlgren, \& Gardner, 2000; Sharif, Rieber, Ozuah, \& Reiber, 2002), as well as an increase in the frequency of book sharing and caregiver enjoyment (Golova, Alario, Vivier, Rodriguez, \& High, 1999).

Book-sharing interventions are typically held in small groups, with a facilitator guiding parents through key lessons and principles. However, researchers have experimented with a number of alternative delivery formats. One that has received considerable interest is dispensing with a facilitator and delivering the intervention entirely on a video. The advantages of using video format are cost efficiency and consistency in delivery of the intervention (Blom- Hoffman, O'Neil- Pirozzi, \& Cutting, 2006). Video formats have proven effective in the delivery of other parenting programs (Webster-Stratton, 1984). This method was first evaluated in relation to book-sharing by Arnold and colleagues (1994). Participants were randomized to a no treatment control group or to a group that received presentation of a training video. This video introduced each of the dialogic reading techniques, described them, and provided examples of these in practice. The children in the intervention group acquired better expressive and receptive language than control group children (Arnold et al., 1994). More recently, the 'Read together talk together' instructional video developed by Whitehurst was evaluated in a small-scale randomized controlled trial (RCT) in Australia. It revealed positive effects on both parental book-sharing techniques and child language (BlomHoffman et al., 2006).

Most research and evaluation of book-sharing interventions has taken place in the U.S. and other high-income countries. There has been very little research on book-sharing 


\section{BOOK-SHARING META-ANALYSIS}

conducted in low and middle income countries (LMICs). The first such trial, a kindergartenbased programme for two-year-old children, was conducted in Mexico (Valdez-Menchaca \& Whitehurst, 1992). Compared to a no intervention control group, children in the intervention group demonstrated significantly better expressive and receptive vocabulary following training, as well as the use of longer and more complex sentences. A second trial carried out in rural Bangladesh evaluated a preschool classroom-based intervention for five year olds, with index children also showing significantly improved expressive vocabulary compared to controls (Opel, Ameer, \& Aboud, 2009). Most recently, two studies conducted in an impoverished urban South African township provided strong evidence for the efficacy of dialogic book-sharing in LMIC settings (Cooper et al., 2014; Vally, Murray, Tomlinson, \& Cooper, 2015). A small pilot RCT (Cooper et al., 2014) of weekly group book-sharing training over eight weeks, with carers of 14 to 18 month olds, showed benefits to carer booksharing skills and to child attention and language outcomes. In a subsequent larger RCT, compared to a control group that received no intervention, the 14-16 month old children whose carers received the dialogic book-sharing training showed significant and substantial improvement in expressive and receptive language, as well as in focal attention (Vally et al., 2015).

\section{Earlier Reviews}

An early meta-analysis by Bus and colleagues (1995), including 29 studies, examined the association between frequency of parent-child book-sharing at home and language development, emergent literacy and reading achievement. This revealed an overall medium effect size of $d=0.59$ on the collective language and literacy outcomes (Bus et al., 1995). These effects were independent of family socioeconomic status. Importantly, however, this review included only cross-sectional observational research and did not consider intervention studies. A more recent meta-analysis of 16 studies, assessed the added value of dialogic 


\section{BOOK-SHARING META-ANALYSIS}

reading and included both quasi-experiments and RCTs (Mol, Bus, de Jong, \& Smeets, 2008). This review reported an overall effect of dialogic reading interventions on child vocabulary outcomes of $d=0.42$, CI: $0.30,0.53$. It also showed, as had the review of observational studies by Bus and colleagues (1995), the importance of child age, with younger, preschool children (2-3 year olds) benefitting more from these parental interventions than older children in kindergarten (4-5 year olds) (i.e. $\mathrm{d}=0.50$ and 0.14 respectively).

\section{Rationale and Objectives}

There has been no systematic review or meta-analysis that has focused exclusively on the findings of RCTs of the efficacy of interactive book-sharing interventions with carers of children. Such a review and analysis would constitute the most scientifically rigorous examination to date of the impact of interactive book-sharing interventions on language development in young children. It has been a decade since the last review of the impact of book-sharing interventions on language development was published (Mol et al., 2008), and several randomized trials have been conducted since then. Further, previous reviews have focussed exclusively on psychological and educational evidence and not searched medical and public health databases, such as Medline and EMBASE. The current review involved a wide search and also expands on previous reviews by considering a novel secondary outcome (caregiver book-sharing competence), and by examining the sources of variation in impact (e.g., intervention dosage). Finally, the present study used robust variance estimation, a relatively novel application for meta-analysing data, which permitted the inclusion of all relevant effect sizes in each meta-analysis. This is an important improvement over past strategies, which either averaged effect sizes within studies, or selected a single outcome. Our method permitted inclusion in the models of all relevant outcome data from each study. 
BOOK-SHARING META-ANALYSIS

The objective of the current systematic review and meta-analysis is to answer the following primary question: To what extent does the evidence from RCTs of shared picture book reading interventions delivered to caregivers support the conclusion of a benefit to child expressive and receptive language outcomes? The review includes consideration of the impact of child age, dosage (brief versus more intense interventions), modality (individual vs group), country income level (High income (HIC) versus LMIC), and parental education (post-school ((tertiary)) vs non-tertiary).

The current review also investigated the extent to which these interventions lead to an improvement in caregivers' book-sharing competence. In other words, with what degree of fidelity are these interventions being delivered by caregivers to their children. This outcome has not previously been considered in meta-analyses, but is worthy of investigation. There has been at least one formal mediation analysis (Murray et al., 2016) that showed how improvements in caregiver book-sharing competence associated with training accounted for much of the improvement in child language. Given that the aim of these interventions is to enhance caregiver book-sharing behavior, we believe that any outcomes measuring this construct should be included in the meta-analysis.

\section{Methods}

\section{Protocol, Registration and Reporting Standards}

A protocol was submitted to the PROSPERO registry for systematic reviews (CRD number: 42017057258) with child language as the primary outcome. While this registered protocol also specified assessment of child socio-emotional outcomes as a secondary outcome, a recent meta-analysis (Xie, Chan, Ji, \& Chan, 2018) focussed directly on this question, rendering this analysis redundant. PRISMA guidelines of reporting were followed.

\section{Eligibility Criteria}


BOOK-SHARING META-ANALYSIS

Types of studies. We included RCTs in which caregiver and child dyads were allocated to intervention or control groups either individually or by cluster. Only English language publications were considered. No publication date, or publication status restrictions were imposed.

Types of participants. The population of interest was defined as children between the ages of one and six years (12-72 months), regardless of language, country income level, race, or other socio-demographic indicators. This specific age group was chosen in line with the findings of previous research that suggested that book-sharing, particularly in the first six years of life, was related to language development, emergent literacy, and reading achievement (Bus et al., 1995). Any primary caregiver of a child (e.g., mother, father, grandmother) was eligible for inclusion for the caregiver competence secondary outcome.

Types of interventions. Studies that evaluated the impact of shared picture book reading interventions that targeted caregivers were eligible for inclusion. Studies were included if they were designed to promote techniques involved in effective book sharing (regardless of whether the intervention was described as 'dialogic reading' or 'interactive reading'). The intervention dose was expected to vary between studies, and both brief interventions and more intensive interventions involving multiple sessions were eligible for inclusion. All modes of delivery were considered, including 'live' face-to-face delivery and video-based interventions. Control conditions were considered that were passive (i.e., no intervention or waiting list) and active (i.e., treatment as usual or a non-book-sharing intervention).

Types of outcome measures. To be eligible for inclusion a study needed to have assessed at least one quantitative outcome of child language. The most common infant and child language assessments used in book-sharing intervention studies were the Peabody Picture Vocabulary Test (PPVT) (Dunn \& Dunn, 1981), the Expressive One Word Picture 
BOOK-SHARING META-ANALYSIS

Vocabulary Test (EOWPVT) (Gardner, 1990) and the MacArthur-Bates Communicative Development Inventory (CDI) (Fenson et al., 2000). However, other language assessment instruments were also considered.

Secondary outcomes were measures of caregiver book-sharing competence. Since this examination was novel and exploratory, no restriction was placed on the nature of the outcome or the method of assessment. Outcomes of caregiver book-sharing competence assess the degree to which the caregivers - to whom the intervention is directed - are able to apply the techniques included in the intervention. This competence is commonly referred to in the literature as book-sharing 'facilitation', or 'sensitivity'.

\section{Search Methods for Identification of Studies}

Information sources. We used a comprehensive search strategy in order to search an extensive range of relevant electronic databases systematically. The search was applied to PsycINFO, and then adapted for each of the following databases: MEDLINE, EMBASE, ERIC, Education abstracts (H.W. Wilson), Cochrane Central Register of Controlled Trials (CENTRAL), and CINAHL. This range of education, psychology, and medical databases provides considerable breadth.

Since initial background reading suggested that there were not many relevant RCTs, we opted for a sensitive literature search in order not to overlook any potentially important studies (Petticrew \& Roberts, 2006).

\section{Search}

The following key concepts were identified: 'Reading', 'Parent or caregiver', 'Randomized Controlled Trial'.

These concepts were expanded to include related terms, synonyms, and subject headings specific to each database. The search strategy made use of multiple sensitive 


\section{BOOK-SHARING META-ANALYSIS}

searches that reflect the different elements of the primary question. Appropriate search options - including Boolean operators, MESH terms, truncation and proximity operators were used to construct and combine searches separately for each of the databases. All final searches were conducted on 22/03/2018. The list of databases searched, details of the number of articles retrieved for each database and the full search strategy for the PsycINFO database are provided in the supplementary material (Table S1).

Reference lists of known RCTs, as well as previous relevant reviews, were scanned for further studies that may have been missed in the initial electronic search. In one case, where a full manuscript could not be located, the author (Towson, 2014) was contacted and provided the necessary material.

\section{Study Selection}

All identified records were exported into EndNote reference manager after which duplicates were removed. Articles were independently screened by two researchers (ND and LH) and sequentially excluded according to the eligibility criteria. Initially titles were screened to reduce the number of initial records, and then the abstracts of the remaining records were scanned to confirm appropriateness. Following this, full text publications of the remaining studies were obtained and read by the same two researchers in order to make final decisions on whether to include or exclude them. Any disagreements between them over the eligibility of particular studies were resolved through discussion with a third reviewer (PC).

\section{Data Collection Process}

A tailored data extraction form (based on the Cochrane Handbook recommendations) was developed in order to obtain all the necessary information from the included studies. Two reviewers (ND and $\mathrm{LH}$ ) extracted data independently into excel and discrepancies were identified and resolved through discussion with a third reviewer (GM). 
BOOK-SHARING META-ANALYSIS

\section{Data Items}

We extracted data from included studies on the following key dimensions: characteristics of the trial setting and population, inclusion and exclusion criteria, baseline characteristics of participants, research design and analysis methods, type and details of intervention, type of outcome measures, risk of bias, results of interventions on relevant outcomes.

\section{Risk of Bias in Individual Studies}

The Cochrane Handbook (Higgins \& Green, 2011) risk-of-bias tool was applied to all included studies. The tool ranks each risk of bias domain as 'high', 'low', or 'unclear' risk. The domains of risk reported are: random sequence generation, allocation concealment, blinding of participants, blinding of outcome assessment, handling of incomplete outcome data, and selective reporting of outcomes. Two reviewers assessed each study for risk of bias (ND and $\mathrm{LH}$ ) and any discrepancies were resolved through discussion involving a third reviewer (PC).

\section{Data Preparation and Summary Measures}

For all included studies we computed the standardized mean difference $(d)$ and the 95\% confidence intervals for measures of expressive and receptive language and caregiver competence. These $d$ values and 95\% CIs were entered into Microsoft Excel and the standard error (SE) was obtained according to the Cochrane Handbook Guidelines (Higgins \& Green, 2011). To account for the design effect, the sample size of cluster-randomized trials was reduced to the effective sample size by using the intra-cluster correlation coefficient. Details of the data transformations are provided in the Supplemental materials.

\section{Analysis}


BOOK-SHARING META-ANALYSIS

A robust variance estimation meta-analysis model was chosen as the most suitable approach for the analysis to account for multiple effect sizes per study per outcome, or multiple intervention arms (Hedges, Tipton, \& Johnson, 2010). This approach allows for the inclusion of any number of dependent effect size estimates within a single analysis. For example, it is possible to include within a single analysis multiple measures of expressive language from multiple follow-up points from a study with multiple intervention arms. This approach meant that we did not ignore any appropriate outcomes, or make arbitrary decisions about the 'most appropriate' outcome that matched our criteria, or create synthetic average effect sizes within single studies (Tanner- Smith \& Tipton, 2014).

Expressive language, receptive language and caregiver book-sharing competence were all analysed separately. Meta-analysis was conducted using Stata 15 software and package -robumeta (Hedberg, 2011).

To assess risk of publication bias, we plotted each trial's effect against the standard errors (Sterne et al., 2011), creating a single effect size per study per outcome. Where there were multiple outcomes per study, the effect sizes and variances were combined (Borenstein, Hedges, Higgins, \& Rothstein, 2009). These were then represented as Egger's plots through the Stata metabias command and output. We used Egger's regression asymmetry tests to quantify any visible bias (Egger, Smith, Schneider, \& Minder, 1997).

\section{Additional Analyses}

We planned to conduct five pre-specified subgroup analyses on primary outcomes, provided that there were sufficient studies available for a meta-regression analysis. These were intervention dosage, child age, parental education, modality (individual vs group based), and country-income level (HIC vs LMIC) (World Bank, 2018).

To examine the impact of dose on the outcome variables, a binary variable for intervention intensity was created based on the distribution of durations: 'low intensity (i.e., 


\section{BOOK-SHARING META-ANALYSIS}

less than 60 minutes of contact time), and 'medium to high intensity' (i.e. more than 60 minutes), corresponding closely to a median split of intensity for the 19 studies. Second, studies were categorised according to parental education level because of previous reports that parental education may bear on whether the intervention is delivered to the child with fidelity (Mol et al., 2008). A binary variable was created: studies were grouped according the whether a majority (>50\%) of parents had, or did not have, some form of tertiary education. For child age, based on indications that intervening early in a child's life is associated with greater improvement in child outcome than later intervention (Mol et al., 2008), child age was dichotomized at the most common point of preschool attendance: those with mean child age at base-line of less than 36 months (younger) and those with mean child age of 36 months or greater (older). Finally, for the modality moderator analysis, only direct instruction (faceto-face) interventions were considered. Interventions that consisted of only video or phone based intervention $(n=3)$ were excluded from this analysis.

\section{Results}

\section{Study Selection}

The results of the systematic search are documented in a PRISMA flow diagram in Figure 1 below. In total, the search yielded 6,448 eligible studies, after duplicates had been removed. Of these, 6,411 were identified via the electronic search and a further 37 by hand searching the reference lists of the two relevant prior reviews and meta-analyses (Bus et al., 1995; Mol et al., 2008) as well as recent evaluation studies. The 6,411 studies were then screened for eligibility and 254 potentially eligible studies were identified. The abstracts of these studies were then read and in the process, 201 studies were discarded because they did not meet the inclusion criteria. This produced a final list of 53 studies for which full text articles were sourced. A reading of these 53 full text studies led to 33 being excluded for reasons listed in the flow diagram. 
BOOK-SHARING META-ANALYSIS

INSERT FIGURE 1 ROUGHLY HERE

\section{Study Characteristics}

The search yielded a total of 19 RCTs (from 20 papers) that met the inclusion criteria. The study characteristics appear in Table 1 . Of the included studies, 11 were conducted in the United States, while the remaining eight studies were distributed across other high-income countries (including two each in Hong Kong and Australia) and middle-income countries (South Africa (two), Brazil, Turkey). The included studies varied according to length of intervention (dosage), participant characteristics, intervention setting, intervention modality, and specific outcomes measured.

\section{Outcomes Measured}

All language outcomes measured were categorised as either 'expressive' or 'receptive'. Table S2 of the supplementary materials provides details of all the language measures used in the included studies, as well as the languages in which they were used. The two most common measures of expressive language were the Expressive One Word Picture Vocabulary Test (EOWPVT) and the verbal subset of the Illinois Test of Psycholinguistic Abilities (ITPA). One study (Sim, Berthelsen, Walker, Nicholson, \& Fielding-Barnsley, 2014) used the Hundred Picture Naming Test (HPNT) (Fisher \& Glenister, 1992) for expressive language outcomes. The MacArthur-Bates Communicative Development Inventory (CDI) was used in three studies with young children as a measure of expressive language (Cronan, Cruz, Arriaga, \& Sarkin, 1996; High et al., 2000; Vally et al., 2015). One study (Blom- Hoffman et al., 2006) used child on-task verbalizations during shared reading as the measure of expressive language. Two studies used standardized measures of Mean Length of Utterance (MLU) as a measure of expressive language (Dale, Crain-Thoreson, Notari-Syverson, \& Cole, 1996; Huebner, 2000), with the latter also using total utterances, 


\section{BOOK-SHARING META-ANALYSIS}

and variety of words used during book-sharing as measures of expressive language. Receptive language outcomes were predominantly measured with the Peabody Picture Vocabulary Test (PPVT). Goldfeld and colleagues (2012) made use of the Clinical Evaluation of Language Fundamentals, Australian edition (CELF-P2). Cronan and colleagues (1996) measured receptive language using the PRIMER language comprehension book . Outcome data on caregiver competence were reported in six studies. The variables that represented this outcome included 'facilitating verbalizations' (Blom- Hoffman et al., 2006), caregiver 'expansions and follow-up questions' (Dale et al., 1996), book-sharing 'sensitivity' and 'reciprocity' (Cooper et al., 2014; Murray et al., 2016) and 'interactive reading style' from the Adult/Child Interactive Reading Inventory (Weisleder et al., 2017).

\section{Intervention Dose}

There was considerable variation in the dose of the included interventions. Some studies were minimal and consisted, for example, of a nurse spending approximately five minutes during well-child visits discussing shared reading with a mother (Goldfeld et al., 2012). Other interventions were far more intensive. For example, Cronan and colleagues (1996) administered an 18-session programme to mothers in their homes. Just over half of the interventions (i.e. 10 out of the 19 studies) involved multiple sessions with training time in excess of 60 minutes with caregivers.

\section{Participant Characteristics}

The included studies involved 2,594 participating infant-caregiver dyads. Child age at the start of the studies ranged from 8.85 months (Goldfeld et al., 2012) to 66 months (Sim et al., 2014). All studies reported on typically developing children only, apart from Dale (1996) and Towson (2014), who included children with mild to moderate language delays and with special education needs respectively. Reporting varied widely with regard to socio- 
BOOK-SHARING META-ANALYSIS

demographic variables, which precluded the construction of a socioeconomic status variable (but the data indicated that socio-economic status was essentially co-extensive with parental education, which was more reliably reported). Some studies were conducted with populations that had a middle-class profile (e.g., (Sim et al., 2014; Whitehurst et al., 1988), while others included predominantly low-income households living in areas characterized by high poverty and deprivation (e.g., (Cronan et al., 1996; Vally et al., 2015). The education levels of the caregivers in the studies also varied considerably. For the purpose of examining the impact on child outcome of carer education level, the latter was dichotomized as majority postschool educated or not. According to this categorization, eight studies had caregivers with majority tertiary education and 10 studies without this level of caregiver education. One study did not report any information on caregiver education level (Dale et al., 1996). INSERT TABLE 1 ROUGHLY HERE

\section{Intervention Modality}

The majority of interventions $(k=15)$ were carried out in-person. These were either conducted one-on-one (e.g., (Cronan et al., 1996), or in a small group (e.g., (Vally et al., 2015)). Three of the interventions were administered in video format. For example, BlomHoffman and colleagues (2006) made use of the "Read together talk together" dialogic reading instructional video developed by Whitehurst (2002). One study administered the intervention in a 20-minute instructional phone call (Wing-Yin Chow \& McBride-Chang, 2003).

\section{Risk of Bias within Included Studies}

The Cochrane Risk of Bias Tool was used to evaluate the risk of bias within the included studies. 


\section{BOOK-SHARING META-ANALYSIS}

Only four studies provided sufficient information on how random sequence generation was handled and qualified as low risk for this dimension. Allocation concealment was often not reported in sufficient detail: 10 studies were judged as having an unclear risk of bias. Blinding of participants was largely unproblematic for the purposes of the current review, as young children or infants were being assessed and were not aware of the intervention their parents had received. However, for studies that used parent self-report measures of child language (Cronan et al., 1996; Vally et al., 2015), blinding was a potentially important issue because knowledge of having received the intervention could have biased responses. Outcome assessment was generally well blinded, with the majority of studies $(k=14)$ having blind assessors. Four studies were at high risk of bias with respect to outcome assessment, as the assessors were not sufficiently blinded to group allocation. Attrition and incomplete outcome data represented a source of bias in two of the included studies (Arnold et al., 1994; Lonigan \& Whitehurst, 1998). One study provided insufficient information to determine whether missing data could have been problematic (Arnold et al., 1994), while another considered data only from participants who had completed post-test assessments, even though there had been substantial drop out (Lonigan \& Whitehurst, 1998).

\section{Synthesis of Results}

Results of all effect sizes from included studies for expressive and receptive language, and for caregiver competence outcomes, are presented in forest plots in Figures 2 and 3. For overall expressive language outcomes, 16 studies provided a total of 35 effect sizes with data from 1664 children. The forest plots show the study with which each effect size is associated, and report the standardized mean difference, and 95\% confidence intervals. The forest plot also provides the combined weighted effect of the outcomes. The pooled analysis suggested evidence $(p<0.001)$ for interactive book sharing having a small sized effect on improving expressive vocabulary when compared to controls $(d=0.41$, CI: $0.20,0.61$; df: 13.44$)$; tau- 
squared $=0.11$. However, there was evidence of considerable heterogeneity between studies $\left(\mathrm{I}^{2}=72 \%\right)$.

\section{INSERT FIGURE 2 ROUGHLY HERE}

For overall receptive language outcomes, 16 of the studies provided a total of 24 effect sizes on data from 1871 children. The results of the meta-analysis are provided in the forest plot in Figure 3. The pooled result of the robust variance estimation meta-analysis suggested evidence $(p=0.001)$ for interactive book-sharing interventions having a small sized effect in improving receptive vocabulary when compared to controls $(d=0.26$; CI: $0.12,0.40$; df: 11.28); tau-squared $=0.03$. There was evidence of some heterogeneity for this outcome $\left(\mathrm{I}^{2}=54 \%\right)$.

\section{INSERT FIGURE 3 ROUGHLY HERE}

For overall caregiver competence outcomes, 6 of the studies provided a total of 15 effect sizes on data from 481 caregivers. The results of the robust effects meta-analysis are provided in the forest plot in the supplementary material (Figure S2). The pooled result of the robust variance estimation meta-analysis provided evidence $(p=0.009)$ for interactive book sharing interventions having a large sized effect in improving caregiver book sharing competence when compared to controls ( $d=1.01$; CI: $0.40,1.63 ;$ df: 4.79$)$; tau-squared $=0.29$. For this outcome there was also considerable evidence of heterogeneity $\left(\mathrm{I}^{2}=81 \%\right)$.

\section{Risk of Bias Across Studies}

The potential risk posed by publication bias was assessed by way of Egger's regression plots. These were generated by plotting the sets of standard normal deviates of effect estimates for all outcomes against their standard errors. Studies with multiple effect sizes per outcome were combined for this process by following the method proposed by Borenstein and colleagues (2009). This produced a single effect size and standard error per study per outcome that was used for the funnel plots, provided in the supplementary material 


\section{BOOK-SHARING META-ANALYSIS}

(Figures S3 - S5). The examination of the Egger's plots revealed low asymmetry, representing low risk of bias across studies for all three outcomes. This was confirmed by non-significant results on the Egger's tests. This held across all three outcomes: expressive language $(t=1.73 ; p=0.11)$, receptive language $(t=1.22 ; p=0.24)$, and caregiver competence $(t=2.08 ; p=0.11)$.

\section{Additional Analyses}

Five subgroup meta-regression analyses were pre-specified. Analyses according to child age, caregiver education level, modality, and intervention dose were estimated on expressive and receptive language outcomes; and country income level (HIC vs LMIC) moderator analysis was estimated on receptive language only due to insufficient data on expressive language.

There was no clear evidence that child age or caregiver education moderated the effect of the intervention on either expressive or receptive language outcomes, and there was no evidence that country income level moderated the effect of the intervention on receptive language. For clarity, moderator analyses were also run with age as a continuous variable (in years). These produced very similar non-significant results.

There was weak evidence $(p=0.07)$ that medium to high intensity interventions were associated with improved expressive language when compared with low intensity $(B=0.33$; CI: -0.04, 0.71). For receptive language outcomes, medium to high intensity interventions were also associated with somewhat better outcomes $(B=0.21$; $C I$ : $-0.07,0.49)$, however, this relation was not significant $(p=0.11)$. For those receiving medium to high intensity interventions, the effect size for expressive language was $d=0.54$ (CI: $0.26,0.82 ; p=0.002$ ), whereas for those receiving the low intensity interventions it was $d=0.19$ (CI:-0.10, 0.50; $p=0.15$ ). Similarly, for those receiving medium to high intensity interventions, the effect size 
BOOK-SHARING META-ANALYSIS

for receptive language was $d=0.34$ (CI: $0.17,0.50, p=0.002)$ : and for those receiving low intensity interventions it was $d=0.09$ (CI: $-0.25,0.45 ; p=0.37)$.

For modality, there was evidence $(\mathrm{p}<0.01)$ that, compared to individually delivered interventions, group-based interventions were associated with more improvement in both expressive language $(B=0.47 ; C I: 0.17,0.77 ; p<0.01)$ and receptive language $(B=0.30 ; C I$ : $0.06,0.54 ; \mathrm{p}=0.02)$. These findings should, however, be interpreted with caution as the degrees of freedom for both models were below the advised level. For clarity, we ran an additional sensitivity analysis where we removed from the analysis the study of individually delivered training by Goldfeld (2012), as it was the study with the largest sample size, but delivered the most minimal intervention. The results when this study was excluded did not alter the overall pattern of the findings, however the result for receptive language was no longer statistically significant $(\mathrm{p}=0.12)$.

INSERT TABLE 2 ROUGHLY HERE

\section{Discussion}

\section{Summary of Evidence}

The current systematic review aimed to assess the impact of dialogic book-sharing interventions on the language development of young children by appraising and synthesizing evidence from all identified RCTs. Overall, evidence of an acceptable quality suggested that, compared to controls, shared reading interventions targeted at parents have a small positive effect on both the expressive $(d=0.41)$ and receptive language $(d=0.26)$ of children. When only the 10 studies with longer duration of intervention (i.e., over 90 minutes) were considered, these effect sizes were $d=0.54$ and $d=0.34$ respectively. The better outcome for expressive language compared to receptive language could be a function of the fact that a central feature of book-sharing interventions is encouraging the child to take an active verbal role in the activity (Mol et al., 2008). Many of the dialogic techniques employed seek to 


\section{BOOK-SHARING META-ANALYSIS}

elicit a verbal response and this promotion of child verbal participation may account for the consistent benefit in child expressive language. However, recent research using confirmatory factor analysis on multiple measures of language, suggests that expressive and receptive vocabulary are best conceptualised as a single construct (Lonigan \& Milburn, 2017). Thus, the differences in effects found may be a result of variability in how these skills were measured, rather than reflecting any actual differences in the children's capacities.

Measures of improvement in caregiver book-sharing competence provide a means of understanding how improvement in child language might be effected. Such measures reveal the extent to which the techniques and principles taught to parents during the interventions have been adopted and implemented. Unfortunately, few studies reported on such outcomes, which precluded a formal subgroup analysis of implementation fidelity. However, for the six studies that did provide relevant data, a large effect size $(d=1.01)$ was observed for the pooled effect on caregiver book-sharing competence.

While this is encouraging, only one study reported the appropriate meditational analysis to establish the impact of book-sharing competence on child language. Murray and colleagues (2016) found that improvements in caregiver sensitivity during book-sharing significantly mediated the impact of the intervention on receptive language, while improvements in caregiver-child reciprocity during book-sharing mediated the impact of the intervention on both child expressive language and receptive language (and child focal attention).

Contrary to an earlier suggestion (Mol et al., 2008), we did not find child age to be related to improvements in either expressive or receptive language: the extent to which language skills benefitted was no different for those younger or older than 3 years. Similarly, whether carers did or did not have post-school education did not bear on child language outcomes. This is especially important as it suggests that caregiver directed book-sharing 


\section{BOOK-SHARING META-ANALYSIS}

interventions can be successful across the full range of parental education, even when there has not previously been a routine of book-sharing (as is the case in families with less educated parents (Bus et al., 1995; Mol et al., 2008)).

The next subgroup analysis aimed to determine whether the effect of the intervention varied across different populations by comparing studies in countries with different income levels (LMIC and HIC). This is a particularly important question, given that the burden of early developmental and language deficit falls in LMIC - predominantly in Sub-Saharan Africa and Asia (Black et al., 2017). There was no evidence for country income level having any association with child receptive language: the interventions were just as effective in LMIC as they were in HIC. Indeed, the model somewhat favoured LMICs $(B=-0.15)$. There was insufficient data to explore this issue in relation to expressive language. Given that, relative to other more comprehensive ECD interventions, book-sharing programs are inexpensive and can be easily delivered, it is surprising that so few evaluations of booksharing have been carried out in LMICs. Further work of this nature is needed to establish the case for scale up of this intervention in LMIC settings. However, the current analysis provides important preliminary evidence for the global applicability of these interventions.

Intervention dosage was revealed to be an important factor in the current analysis. The categorization into low and high dosage was necessarily crude $(<60$ minutes vs $>60$ minutes of collective intervention time) but it was, nevertheless, instructive: children benefitted significantly more in their expressive language when their caregivers had taken part in a more intensive intervention; and there was a trend for the same association for child receptive language. Indeed, the brief interventions had no significant impact on child language outcomes. Brief interventions do not therefore appear to be an efficient use of resources, possibly because they fail to effect the necessary change in caregiver book-sharing behavior. 
BOOK-SHARING META-ANALYSIS

A potentially important finding was that group-based interventions were more effective than one-on-one interventions for both child language outcomes. A possible explanation is that the group format offers more social support to participating caregivers. Indeed, many of the group-based interventions incorporated opportunities for parents to share their experiences of book-sharing with their children at home as well as discuss perceived barriers and challenges they were experiencing (e.g. Vally et al., 2015; Weisleder et al., 2018). However, these findings should be interpreted with caution, given the limited number studies that used a one-on-one format and the fact that that two of these five studies had very low intensity interventions (Goldfeld et al., 2012; High et al., 2000).

\section{Strengths and Limitations}

The current study is the first systematic review and meta-analysis of caregiverdirected book-sharing interventions focusing specifically on randomized trials. It involved a wide-ranging search of educational, psychological, medical, and public-health databases. We used a novel statistical approach, namely, robust variance estimation. This allowed for multiple effect sizes per study per outcome to be incorporated, thus providing a more precise estimation than previously achieved using the pooled effect of each outcome (Tanner- Smith \& Tipton, 2014). We also went beyond investigating the effect of these interventions on child language by also investigating their effect on caregiver book-sharing behaviors.

A key limitation of the included studies was the paucity of follow-up assessment of children. While all studies included both pre-intervention and immediate post-interventions assessments, few provide any findings on outcome beyond the immediate post-intervention assessment. This leaves unanswered the question of the durability of effects. The language and pre-literacy skills that interactive book-sharing interventions help to build are only fully realized when a child enters the schooling system and begins to engage with formal literacy instruction. As such, it is of critical importance that a longitudinal study design is employed 


\section{BOOK-SHARING META-ANALYSIS}

to establish whether the impressive short-term benefits for child language are evident at the point of school entry. The only study to follow up with children more than a year following the intervention was Goldfeld and colleagues (2012) who found no long term impact of the intervention; however, this study involved the briefest intervention of all studies included in this review, and it produced one of the smallest post-intervention effects on child language, so an absence of a long-term impact so an absence of a long-term impact cannot be regarded as a valid reflection of the long-term impact of dialogic book-sharing.

A second limitation is the reporting on implementation fidelity. Such reporting was a rarity in the studies reviewed. Our understanding of the mechanisms responsible for intervention effects would benefit considerably if studies consistently collected data on parents' application of learned techniques. This would enable mediator analyses, of the sort reported by Murray and colleagues (2016), which could elucidate core components that drive intervention effects. Such analysis could serve the cause of distilling a parsimonious intervention package that could potentially improve efficiency and increase reach.

A third limitation was that many of the included studies lacked information about the random sequence generation used $(k=12)$ and about allocation concealment $(k=10)$. These two aspects of study execution are central to the RCT design, and hence uncertainty regarding how they were carried out means that further caution in interpreting the findings is warranted.

A fourth limitation is that a wide variety of language measures were used in the included studies. Although almost all of these were standardized and well validated instruments, this variability in assessment introduced heterogeneity into the results.

Finally, clinical and statistical heterogeneity were present within the set of included studies. The interventions, while all sharing certain common messages to help parents optimise shared reading practice, came in a variety of forms, which meant that there was a 
BOOK-SHARING META-ANALYSIS

degree of clinical heterogeneity present, reflecting this variability. Further, despite exploration (by means of subgroup analyses) the observed statistical heterogeneity for all three outcomes remain somewhat unexplained.

\section{Implications and Conclusions}

Implications for policy and practice. The current meta-analysis of RCTs on booksharing interventions demonstrates the promise of such interventions for enhancing child language development, at least in the short term. These interventions show efficacy across a variety of settings and modalities, which is encouraging for taking them to scale. Indeed, a strong argument could be made that book-sharing should be considered for any programme that seeks to support early literacy and language development in infants and young children. It is especially significant that the current analysis indicates that book-sharing interventions can be equally effective when targeted at caregivers with low and high levels of education. It is also notable that, contrary to previous suggestions, the current analysis indicates that young and older pre-school children benefit equally from these interventions. Finally, this analysis suggests that there is a dose effect, with brief interventions being unlikely to result in improvements in children's language abilities. Interventions involving multiple sessions, on the other hand, with extended contact time between the instructor and caregiver, are highly likely to result in improvements to child language.

Implications for research. A total of 19 RCTs were identified that met the inclusion criteria. This is a relatively small number of studies. More than half were conducted in the United States, which further limits generalizability. Certain shortcomings in the evidence base emerged through this review and future research should look to address these. First, studies need to include measures of implementation fidelity measuring parents' application of the interactive techniques when book-sharing with their children. This is important for both theory development and intervention refinement. Second, there is a need for longitudinal 
BOOK-SHARING META-ANALYSIS

research into the durability of intervention effects - ideally following children from early childhood to the point of school entry. Third, given the tentative evidence that group-based interventions are more effective than one-on-one interventions, a direct comparison of these two delivery formats in an RCT format could be a valuable addition to this body of literature. Fourth, the studies in this review primarily included only typically developing children. Interventions targeting children with language deficits or children 'at risk', such as those from LMICs, could be an important direction for future research. Finally, and arguably most critically, there is a need for more research in LMIC settings where it is estimated that in excess of 250 million children are at risk of failing to meet their developmental potential (Black et al., 2017). In these settings, interactive book-sharing interventions have the potential to bring about marked, positive effects on children's early language development and, possibly, their wider developmental progress. 


\section{References}

References marked with an asterisk indicate studies included in the meta-analysis.

*Arnold, D. H., Lonigan, C. J., Whitehurst, G. J., \& Epstein, J. N. (1994). Accelerating language development through picture book reading: replication and extension to a videotape training format. Journal of Educational Psychology, 86(2), 235. doi:http://dx.doi.org/10.1037/0022-0663.86.2.235

Berkule, S. B., Dreyer, B. P., Huberman, H. S., Fierman, A. H., \& Mendelsohn, A. L. (2007). Attitudes about shared reading among at-risk mothers of newborn babies. Ambulatory Pediatrics, 7(1), 45-50. doi:https://doi.org/10.1016/j.ambp.2006.10.004

Black, M. M., Walker, S. P., Fernald, L. C., Andersen, C. T., DiGirolamo, A. M., Lu, C., .. . Shiffman, J. (2017). Early childhood development coming of age: science through the life course. The Lancet, 389(10064), 77-90. doi:https://doi.org/10.1016/S0140$\underline{6736(16) 31389-7}$

*Blom - Hoffman, J., O'Neil - Pirozzi, T. M., \& Cutting, J. (2006). Read together, talk together: The acceptability of teaching parents to use dialogic reading strategies via videotaped instruction. Psychology in the Schools, 43(1), 71-78. doi:DOI: 10.1002/pits.20130

Borenstein, M., Hedges, L. V., Higgins, J., \& Rothstein, H. R. (2009). Introduction to metaanalysis: Wiley and sons.

Bornstein, M. H., \& Putnick, D. L. (2012). Cognitive and socioemotional caregiving in developing countries. Child development, 83(1), 46-61. doi:DOI: 10.1111/j.14678624.2011.01673.x

Bus, A. G., Van Ijzendoorn, M. H., \& Pellegrini, A. D. (1995). Joint book reading makes for success in learning to read: A meta-analysis on intergenerational transmission of 
BOOK-SHARING META-ANALYSIS

literacy. Review of educational research, 65(1), 1-21. doi:https://doiorg.ezproxy.uct.ac.za/10.3102/00346543065001001

*Chacko, A., Fabiano, G. A., Doctoroff, G. L., \& Fortson, B. (2017). Engaging fathers in effective parenting for preschool children using shared book reading: A randomized controlled trial. Journal of Clinical Child \& Adolescent Psychology, 1-14. doi:https://doi-org.ezproxy.uct.ac.za/10.1080/15374416.2016.1266648

*Chow, B. W.-Y., McBride-Chang, C., Cheung, H., \& Chow, C. S.-L. (2008). Dialogic reading and morphology training in Chinese children: Effects on language and literacy. Developmental psychology, 44(1), 233. doi:http://dx.doi.org/10.1037/0012$\underline{1649.44 .1 .233}$

*Cooper, P. J., Vally, Z., Cooper, H., Radford, T., Sharples, A., Tomlinson, M., \& Murray, L. (2014). Promoting mother-infant book sharing and infant attention and language development in an impoverished South African population: a pilot study. Early Childhood Education Journal, 42(2), 143-152. doi:https://doiorg.ezproxy.uct.ac.za/10.1007/s10643-013-0591-8

*Cronan, T. A., Cruz, S. G., Arriaga, R. I., \& Sarkin, A. J. (1996). The effects of a community - based literacy program on young children's language and conceptual development. American Journal of Community Psychology, 24(2), 251-272. doi:DOI: 10.1007/BF02510401

*Dale, P. S., Crain-Thoreson, C., Notari-Syverson, A., \& Cole, K. (1996). Parent-child book reading as an intervention technique for young children with language delays. Topics in Early Childhood Special Education, 16(2), 213-235. doi:https://doiorg.ezproxy.uct.ac.za/10.1177/027112149601600206

Dunn, L. M., \& Dunn, L. M. (1981). Manual for the peabody picture vocabulary test-revised. Circle Pines, MN: American Guidance Service. 
BOOK-SHARING META-ANALYSIS

Egger, M., Smith, G. D., Schneider, M., \& Minder, C. (1997). Bias in meta-analysis detected by a simple, graphical test. Bmj, 315(7109), 629-634. doi:https://doiorg.ezproxy.uct.ac.za/10.1136/bmj.315.7109.629

El Moussaoui, N., \& Braster, S. (2011). Perceptions and practices of stimulating children's cognitive development among Moroccan immigrant mothers. Journal of child and family studies, 20(3), 370-383. doi:https://doi-org.ezproxy.uct.ac.za/10.1007/s10826$\underline{010-9401-8}$

Fenson, L., Pethick, S., Renda, C., Cox, J. L., Dale, P. S., \& Reznick, J. S. (2000). Short-form versions of the MacArthur communicative development inventories. Applied Psycholinguistics, 21(1), 95-116.

Fisher, J. P., \& Glenister, J. M. (1992). The hundred pictures naming test: Australian Council for Educational Research.

Gardner, M. F. (1990). Expressive One-word Picture Vocabulary Test, Revised: EO-WPVT$R$ : Academic Therapy Publications.

*Goldfeld, S., Quach, J., Nicholls, R., Reilly, S., Ukoumunne, O. C., \& Wake, M. (2012). Four-year-old outcomes of a universal infant-toddler shared reading intervention: The Let's Read trial. Archives of pediatrics \& adolescent medicine, 166(11), 1045-1052. doi:doi:10.1001/archpediatrics.2012.1099

Golova, N., Alario, A. J., Vivier, P. M., Rodriguez, M., \& High, P. C. (1999). Literacy promotion for Hispanic families in a primary care setting: a randomized, controlled trial. Pediatrics, 103(5), 993-997. doi:doi: 10.1542/peds.103.5.993

Hedges, L. V., Tipton, E., \& Johnson, M. C. (2010). Robust variance estimation in meta regression with dependent effect size estimates. Research synthesis methods, 1(1), 3965. doi:DOI: 10.1002/jrsm.5 
BOOK-SHARING META-ANALYSIS

Higgins, J. P., \& Green, S. (2011). Cochrane handbook for systematic reviews of interventions (Vol. 4): John Wiley \& Sons.

*High, P. C., LaGasse, L., Becker, S., Ahlgren, I., \& Gardner, A. (2000). Literacy promotion in primary care pediatrics: can we make a difference? Pediatrics, 105(Supplement 3), 927-934.

*Huebner, C. E. (2000). Promoting toddlers' language development through communitybased intervention. Journal of Applied Developmental Psychology, 21(5), 513-535. doi:https://doi.org/10.1016/S0193-3973(00)00052-6

*Kotaman, H. (2013). Impacts of dialogical storybook reading on young children's reading attitudes and vocabulary development. Reading Improvement, 50(4), 199-204.

Le Roux, A. (2012). The production and use of wordless picture books in parent-child reading: an exploratory study within a South African context. Stellenbosch: Stellenbosch University.

*Lonigan, C. J., \& Whitehurst, G. J. (1998). Relative efficacy of parent and teacher involvement in a shared-reading intervention for preschool children from low-income backgrounds. Early Childhood Research Quarterly, 13(2), 263-290. doi:https://doi.org/10.1016/S0885-2006(99)80038-6

Lonigan, C. J., \& Milburn, T. F. (2017). Identifying the dimensionality of oral language skills of children with typical development in preschool through fifth grade. Journal of Speech, Language, and Hearing Research, 60(8), 2185-2198. doi:10.1044/2017_JSLHR-L-15-0402

Mendelsohn, A. L., Huberman, H. S., Berkule, S. B., Brockmeyer, C. A., Morrow, L. M., \& Dreyer, B. P. (2011). Primary care strategies for promoting parent-child interactions and school readiness in at-risk families: the Bellevue Project for Early Language, 
BOOK-SHARING META-ANALYSIS

Literacy, and Education Success. Archives of pediatrics \& adolescent medicine, 165(1), 33-41. doi:doi:10.1001/archpediatrics.2010.254

Mendelsohn, A. L., Mogilner, L. N., Dreyer, B. P., Forman, J. A., Weinstein, S. C., Broderick, M., . . . Napier, C. (2001). The impact of a clinic-based literacy intervention on language development in inner-city preschool children. Pediatrics, 107(1), 130-134. doi:doi: 10.1542/peds.107.1.130

Mol, S. E., Bus, A. G., de Jong, M. T., \& Smeets, D. J. (2008). Added value of dialogic parent-child book readings: A meta-analysis. Early Education and Development, 19(1), 7-26. doi:https://doi-org.ezproxy.uct.ac.za/10.1080/10409280701838603

Murray, L. (2014). The psychology of babies: how relationships support development from birth to two: Hachette UK.

*Murray, L., De Pascalis, L., Tomlinson, M., Vally, Z., Dadomo, H., MacLachlan, B., .. . Cooper, P. J. (2016). Randomized controlled trial of a book - sharing intervention in a deprived South African community: effects on carer - infant interactions, and their relation to infant cognitive and socioemotional outcome. Journal of Child Psychology and Psychiatry, 57(12), 1370-1379. doi:DOI: 10.1111/jcpp.12605

Ninio, A. (1980). Picture-book reading in mother-infant dyads belonging to two subgroups in Israel. Child development, 587-590.

Ninio, A., \& Bruner, J. (1978). The achievement and antecedents of labelling. Journal of child language, 5(1), 1-15.

Opel, A., Ameer, S. S., \& Aboud, F. E. (2009). The effect of preschool dialogic reading on vocabulary among rural Bangladeshi children. International Journal of Educational Research, 48(1), 12-20. doi:https://doi.org/10.1016/j.ijer.2009.02.008

Petticrew, M., \& Roberts, H. (2006). Sytematic reviews in the social sciences: a practical guide. . Oxford: Blackwell. 
BOOK-SHARING META-ANALYSIS

*Reese, E., Leyva, D., Sparks, A., \& Grolnick, W. (2010). Maternal elaborative reminiscing increases low-income children's narrative skills relative to dialogic reading. Early Education and Development, 21(3), 318-342. doi:https://doiorg.ezproxy.uct.ac.za/10.1080/10409289.2010.481552

Sharif, I., Rieber, S., Ozuah, P. O., \& Reiber, S. (2002). Exposure to Reach Out and Read and vocabulary outcomes in inner city preschoolers. Journal of the National Medical Association, 94(3), 171.

*Sim, S. S., Berthelsen, D., Walker, S., Nicholson, J. M., \& Fielding-Barnsley, R. (2014). A shared reading intervention with parents to enhance young children's early literacy skills. Early child development and care, 184(11), 1531-1549. doi:https://doiorg.ezproxy.uct.ac.za/10.1080/03004430.2013.862532

Snow, C. E., \& Goldfield, B. A. (1983). Turn the page please: Situation-specific language acquisition. Journal of child language, 10(3), 551-569.

Sterne, J. A., Sutton, A. J., Ioannidis, J. P., Terrin, N., Jones, D. R., Lau, J., . . Schmid, C. H. (2011). Recommendations for examining and interpreting funnel plot asymmetry in meta-analyses of randomised controlled trials. Bmj, 343, d4002. doi:https://doiorg.ezproxy.uct.ac.za/10.1136/bmj.d4002

Tanner - Smith, E. E., \& Tipton, E. (2014). Robust variance estimation with dependent effect sizes: practical considerations including a software tutorial in Stata and SPSS. Research synthesis methods, 5(1), 13-30. doi:DOI: 10.1002/jrsm.1091

*Towson, J. A. (2014). Training Head Start parents in dialogic reading to improve outcomes for children. International Journal of Child Health and Human Development, 7(3), 287.

Valdez-Menchaca, M. C., \& Whitehurst, G. J. (1992). Accelerating language development through picture book reading: a systematic extension to Mexican day care. 
BOOK-SHARING META-ANALYSIS

Developmental psychology, 28(6), 1106. doi:http://dx.doi.org/10.1037/0012$\underline{1649.28 .6 .1106}$

*Vally, Z., Murray, L., Tomlinson, M., \& Cooper, P. J. (2015). The impact of dialogic book - sharing training on infant language and attention: a randomized controlled trial in a deprived South African community. Journal of Child Psychology and Psychiatry, 56(8), 865-873. doi:DOI: 10.1111/jcpp.12352

Van Kleeck, A. (1998). Preliteracy domains and stages: Laying the foundations for beginning reading. Journal of Children's Communication Development, 20(1), 33-51. doi:https://doi-org.ezproxy.uct.ac.za/10.1177/152574019802000105

Vygotsky, L. S. (1978). Mind in society: The development of higher psychological processes (M. Cole, V. John-Steiner, S. Scribner \& E. Souberman., Eds.) Cambridge, Mass.: Harvard University Press. (Original manuscripts [ca. 1930-1934]).

Webster-Stratton, C. (1984). Randomized trial of two parent-training programs for families with conduct-disordered children. Journal of Consulting and Clinical Psychology, 52(4), 666.

Weigel, D. J., Lowman, J. L., \& Martin, S. S. (2007). Language development in the years before school: A comparison of developmental assets in home and child care settings. Early child development and care, 177(6-7), 719-734. doi:https://doi.org/10.1080/03004430701379173

*Weisleder, A., Mazzuchelli, D. S., Lopez, A. S., Neto, W. D., Cates, C. B., Gonçalves, H. A., ... Mendelsohn, A. L. (2017). Reading Aloud and Child Development: A ClusterRandomized Trial in Brazil. Pediatrics, e20170723. doi:doi: 10.1542/peds.2017-0723 Wells, G. (1985). Language development in the pre-school years (Vol. 2): CUP Archive. Whitehurst, G. J. (Writer). (2002). Read together, talk together [Motion picture]: Pearson Early Learning. 
BOOK-SHARING META-ANALYSIS

Whitehurst, G. J., Epstein, J. N., Angell, A. L., Payne, A. C., Crone, D. A., \& Fischel, J. E. (1994). Outcomes of an emergent literacy intervention in Head Start. Journal of Educational Psychology, 86(4), 542. doi:DOI: 10.1037//0022-0663.86.4.542

*Whitehurst, G. J., Falco, F. L., Lonigan, C. J., Fischel, J. E., DeBaryshe, B. D., ValdezMenchaca, M. C., \& Caulfield, M. (1988). Accelerating language development through picture book reading. Developmental psychology, 24(4), 552. doi:DOI: $10.1037 / 0012-1649.24 .4 .552$

*Wing-Yin Chow, B., \& McBride-Chang, C. (2003). Promoting language and literacy development through parent-child reading in Hong Kong preschoolers. Early Education and Development, 14(2), 233-248. doi:DOI: 10.1207/s15566935eed1402_6

World Bank. (2018). World Bank Country Classification by Income. Retrieved from: https://datahelpdesk.worldbank.org/knowledgebase/articles/906519-world-bankcountry-and-lending-groups

Xie, Q.-W., Chan, C. H., Ji, Q., \& Chan, C. L. (2018). Psychosocial effects of parent-child book reading interventions: a meta-analysis. Pediatrics, 141(4), e20172675. 
BOOK-SHARING META-ANALYSIS

Table 1

Characteristics of included studies

\begin{tabular}{|c|c|c|c|c|c|c|c|c|c|c|c|c|}
\hline Author & Year & Country & Population & $\begin{array}{l}\mathrm{N} \text { (int, } \\
\text { control) }\end{array}$ & $\begin{array}{l}\text { Child } \\
\text { age } \\
\text { (mo.) } \\
\end{array}$ & $\begin{array}{l}\text { Parental } \\
\text { education }\end{array}$ & $\begin{array}{l}\text { Baseline } \\
\text { language } \\
\text { control } \\
\end{array}$ & $\begin{array}{l}\text { Dosage } \\
\text { intensity }\end{array}$ & Modality & Control & $\begin{array}{l}\text { Time } \\
\text { between } \\
\text { assessments }\end{array}$ & $\begin{array}{l}\text { No. of } \\
\text { sessions } \\
(\mathrm{min} / \mathrm{session})\end{array}$ \\
\hline Arnold & 1994 & U.S & $\begin{array}{l}\text { Middle } \\
\text { sample }\end{array}$ & $41(24,27)$ & 29 & Tertiary & $\mathrm{Y}$ & Low & Video & No intervention & 4 weeks & $2(15 \mathrm{~min})$ \\
\hline $\begin{array}{l}\text { Blom- } \\
\text { Hoffman }\end{array}$ & 2006 & U.S & $\begin{array}{l}\text { Mixed } \\
\text { sample }\end{array}$ & $18(8,10)$ & 41 & Tertiary & $\mathrm{N}$ & Low & Video & Books & $6 / 12$ weeks & $1(15 \mathrm{~min})$ \\
\hline Chacko & 2017 & U.S & $\begin{array}{l}\text { Low income, } \\
\text { Head Start }\end{array}$ & $126(64,62)$ & 55 & Non-tertiary & $\mathrm{N}$ & Medium to high & Group & No intervention & 10 weeks & 8 (90 min) \\
\hline Chow & 2003 & Hong Kong & Middle class & $54(27,27)$ & 60 & Non-tertiary & $\mathrm{Y}$ & Low & Telephone & No intervention & 8 weeks & $1(20 \mathrm{~min})$ \\
\hline Chow & 2008 & Hong Kong & Middle class & $73(37,36)$ & 63 & Tertiary & $\mathrm{Y}$ & Medium to high & Group & Books & 12 weeks & $1(60 \min )+6^{a}$ \\
\hline Cooper & 2014 & $\begin{array}{l}\text { South } \\
\text { Africa }\end{array}$ & Low income & $25(13,12)$ & 16 & Non-tertiary & $\mathrm{Y}$ & Medium to high & Group & Play intervention & 8 weeks & $7(60 \mathrm{~min})$ \\
\hline Cronan & 1996 & U.S & $\begin{array}{l}\text { Low income, } \\
\text { Head Start }\end{array}$ & $152(83,69)$ & 28 & Non-tertiary & $\mathrm{Y}$ & Medium to high & Individual & No intervention & 28 weeks & $18(30 \mathrm{~min})$ \\
\hline Dale & 1996 & U.S & Middle class & $30(15,15)$ & 61 & N/A & $\mathrm{Y}$ & Medium to high & Group & $\begin{array}{l}\text { Conversational } \\
\text { training }\end{array}$ & 8 weeks & $2(40 \mathrm{~min})$ \\
\hline Goldfeld & 2012 & Australia & Low income & $410(237,173)$ & 9 & Non-tertiary & $\mathrm{N}$ & Low & Individual & TAU* & 4 years & $4(5 \mathrm{~min})$ \\
\hline High & 2000 & U.S & Low income & $150(75,75)$ & 19 & Non-tertiary & $\mathrm{Y}$ & Low & Individual & TAU* & 1 year & $3(5 \mathrm{~min})$ \\
\hline Huebner & 2000 & U.S & Middle class & $115(79,36)$ & 29 & Tertiary & $\mathrm{Y}$ & Medium to high & Group & Books & 12 weeks & $2(60 \mathrm{~min})$ \\
\hline Kotaman & 2008 & Turkey & Middle class & $40(20,20)$ & 47 & Tertiary & $\mathrm{Y}$ & Medium to high & Group & No intervention & 8 weeks & $1(120 \mathrm{~min})$ \\
\hline Lonigan & 1998 & U.S & $\begin{array}{l}\text { Low income, } \\
\text { day-care } \\
\text { subsidy }\end{array}$ & $43(16,27)$ & 45 & Non-tertiary & $\mathrm{Y}$ & Low & Individual & No intervention & 6 weeks & $2(30 \mathrm{~min})$ \\
\hline Reese & 2010 & U.S & $\begin{array}{l}\text { Low income, } \\
\text { Head Start }\end{array}$ & $20(9,11)$ & 49 & Tertiary & $\mathrm{Y}$ & Low & Individual & No intervention & 1 year & $1(45 \min )+5^{a}$ \\
\hline Sim & 2013 & Australia & Middle class & $49(26,23)$ & 66 & Tertiary & Y & Medium to high & Group & Number learning & $8 / 20$ weeks & $1(15 \mathrm{~min})$ \\
\hline Towson & 2014 & U.S & $\begin{array}{l}\text { Low income, } \\
\text { Head Start }\end{array}$ & $25(13,12)$ & 47 & Non-tertiary & Y & Low & Group & $\begin{array}{l}\text { Positive } \\
\text { parenting }\end{array}$ & 7 weeks & $1(30 \mathrm{~min})$ \\
\hline Vally & 2015 & $\begin{array}{l}\text { South } \\
\text { Africa }\end{array}$ & Low income & $82(45,37)$ & 15 & Non-tertiary & $\mathrm{Y}$ & Medium to high & Group & No intervention & 10 weeks & 8 (90 min) \\
\hline Weisleder & 2018 & Brazil & Low income & $484(232,252)$ & 37 & Non-tertiary & $\mathrm{Y}$ & Medium to high & Group & $\begin{array}{l}\text { Child care } \\
\text { curriculum }\end{array}$ & 36 weeks & 8 (60 min) \\
\hline Whitehurst & 1988 & U.S & Middle class & $29(14,15)$ & 29 & Tertiary & $\mathrm{N}$ & Medium to high & Group & No intervention & 4/40 weeks & $2(35 \mathrm{~min})$ \\
\hline
\end{tabular}

Note. * TAU stands for Treatment as usual and refers to routine 'well child' visits at pediatric clinics; $a=$ reminders 
BOOK-SHARING META-ANALYSIS

Table 2

Moderator Analyses

\begin{tabular}{lccccc}
\hline \multicolumn{1}{c}{ Moderator } & B & 95\% C.I. & Intercept & 95\% C.I. & Tau $^{\mathbf{2}}$ \\
\hline Age & & & & & \\
$\quad$ Expressive & -0.10 & $-0.51,0.31$ & 0.46 & $0.07,0.84$ & 0.14 \\
$\quad$ Receptive & 0.08 & $-0.20,0.36$ & 0.23 & $-0.01,0.45$ & 0.03 \\
$\begin{array}{l}\text { Caregiver Education } \\
\quad \text { Expressive }\end{array}$ & 0.16 & $-0.29,0.61$ & 0.35 & $0.05,0.66$ & 0.11 \\
$\quad \begin{array}{l}\text { Receptive } \\
\text { Country Income Level }\end{array}$ & 0.13 & $-0.12,0.38$ & 0.22 & $0.03,0.42$ & 0.03 \\
$\quad$ Receptive & -0.15 & $-0.63,0.33$ & 0.38 & $-0.24,0.99$ & 0.03 \\
$\quad \begin{array}{l}\text { Intervention Dosage } \\
\quad \text { Expressive }\end{array}$ & $0.33^{+}$ & $-0.04,0.71$ & 0.21 & $-0.10,0.51$ & 0.09 \\
$\quad$ Receptive & 0.21 & $-0.07,0.49$ & 0.12 & $-0.18,0.42$ & 0.02 \\
$\quad \begin{array}{l}\text { Modality } \\
\quad \text { Expressive }\end{array}$ & $0.47^{* *}$ & $0.17,0.77$ & 0.07 & $-0.11,0.25$ & 0.07 \\
$\quad$ Receptive & $0.30^{*}$ & $0.06,0.54$ & 0.06 & $-0.18,0.30$ & 0.02 \\
\hline Note. ${ }^{+} \mathrm{p}<0.1, * \mathrm{p}<0.05, * * \mathrm{p}<0.01$ & & & &
\end{tabular}



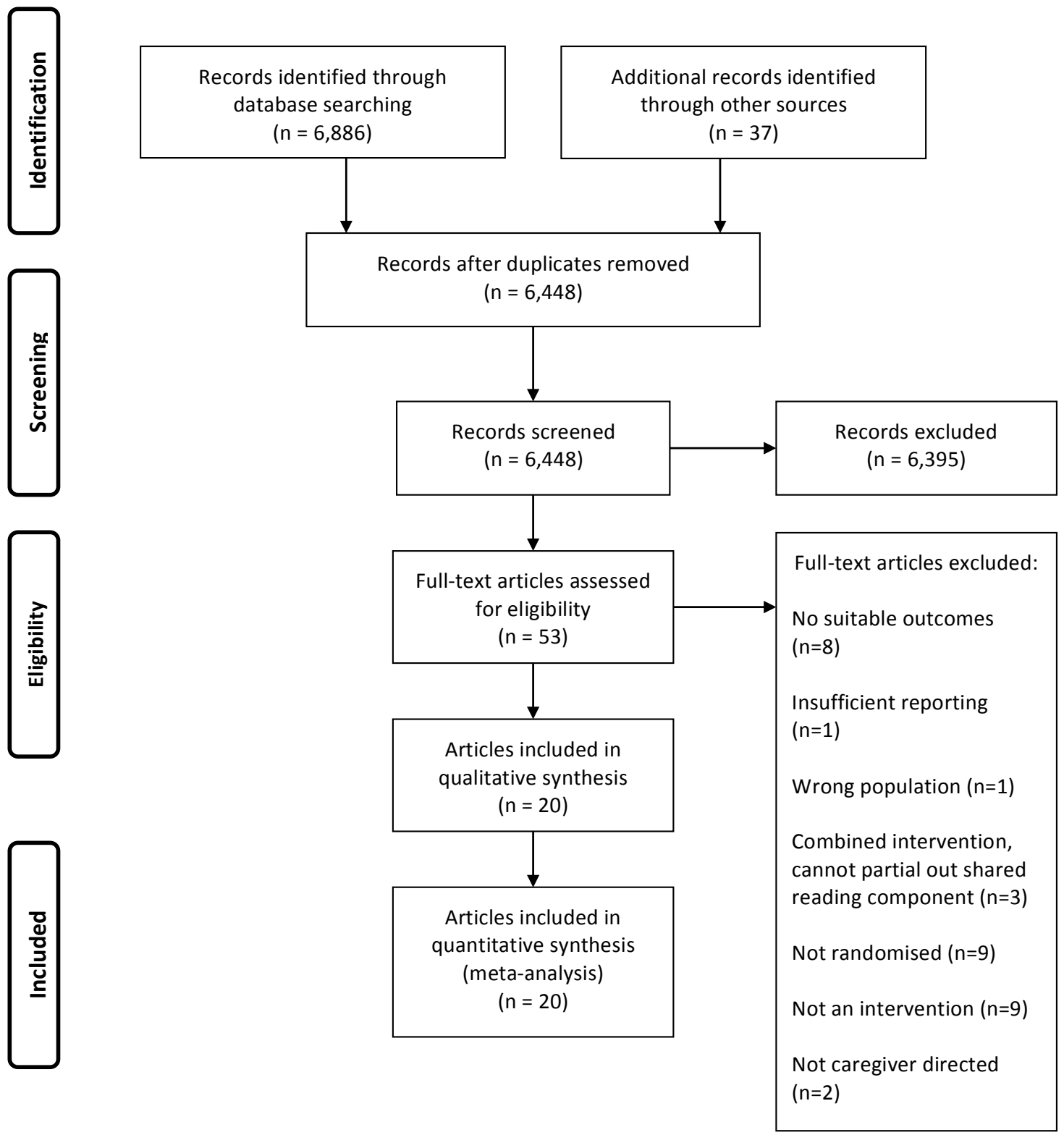

Figure 1: PRISMA flow chart of search results and included and excluded studies 


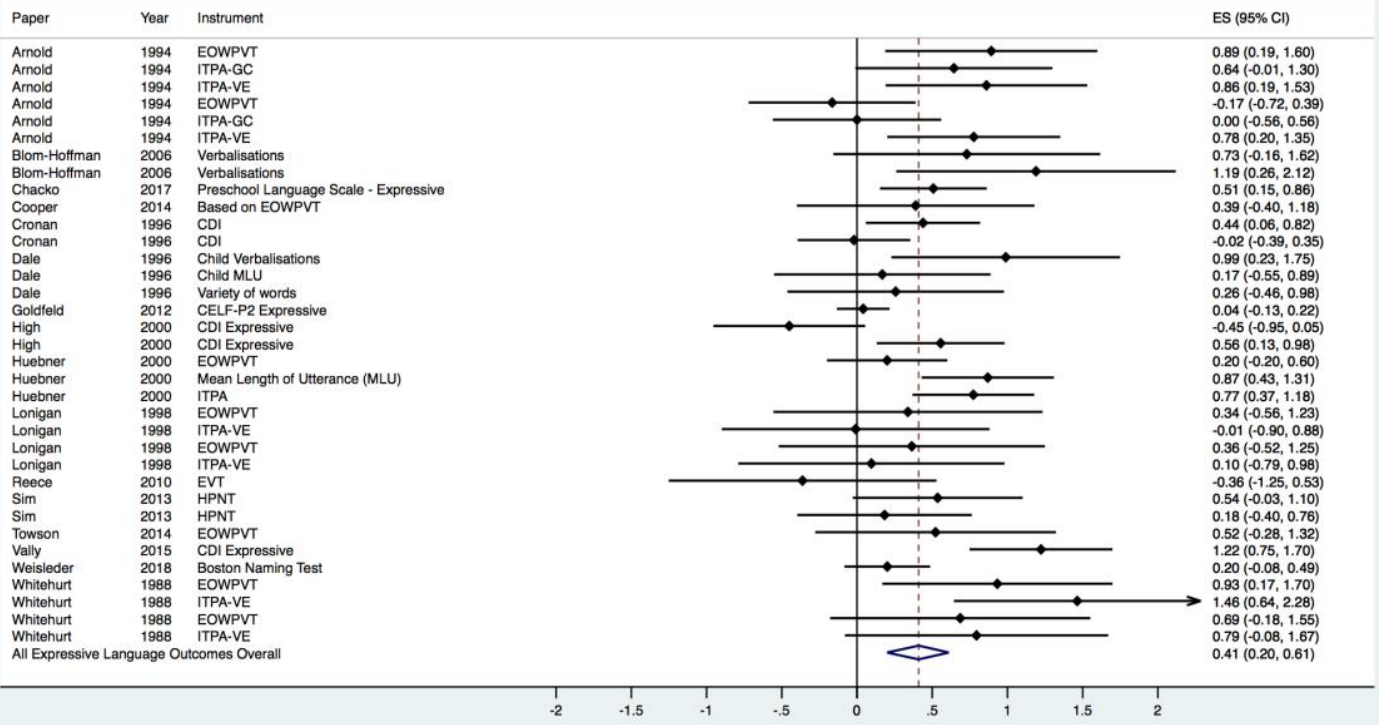

Figure 2: Meta-analysis for expressive language 


\begin{tabular}{lll} 
Paper & Year & Instrument \\
\hline Arnold & 1994 & PPVT \\
Arnold & 1994 & PPVT \\
Chacko & 2017 & Preschool Language Scale - Auditory \\
Chow & 2003 & PPVT \\
Chow & 2003 & PPVT \\
Chow & 2008 & PPVT \\
Chow & 2008 & PPVT \\
Cooper & 2014 & PPVT Adapted \\
Cronan & 1996 & PRIMER \\
Cronan & 1996 & PRIMER \\
Goldfeld & 2012 & CELF-P2 Receptive \\
High & 2000 & CDI Receptive \\
High & 2000 & CDI Receptive \\
Huebner & 2000 & PPVT \\
Kotaman & 2008 & PPVT \\
Lonigan & 1998 & PPVT \\
Lonigan & 1998 & PPVT \\
Sim & 2013 & PPVT \\
Sim & 2013 & PPVT \\
Towson & 2014 & PPVT \\
Towson & 2014 & TVIP \\
Vally & 2015 & CDI Receptive \\
Vally & 2015 & PPVT Adapted \\
Weisleder & 2018 & PPVT \\
Whitehurt & 1988 & PPVT \\
Whitehurt & 1988 & PPVT \\
All Receptive Language Outcomes Overall
\end{tabular}

$0.44(-0.06,0.80)$

$-0.06(-0.84,0.72)$

$0.34(-0.01,0.70)$

$-0.10(-0.47,0.27)$

$0.03(-0.17,0.22)$

$0.23(-0.27,0.73)$

(0.67)

$0.75(0.11,1.39)$

$-0.18(-1.07,0.70)$

$0.33(-0.56,122)$

$0.33(-0.56,1.22)$

$0.12(-0.46,0.70)$

$0.25(-0.54,1.03)$

$0.34(-0.45,1.13)$

$0.98(0.52,1.44)$

$0.32(-0.11,0.76)$

$0.22(0.01,0.43)$

$0.58(-0.17,1.32)$

$0.01(-0.83,0.84)$ $0.26(0.12,0.40)$

Figure 3: Meta-analysis for receptive language 